\title{
Repetition effects and the ordered recall of categorized serial lists'
}

JAMES R. CRAIG and WAYNE H. BARTZ, lowa State University, Ames, Iowa 50010

Two-store memory theories predict no repetition effects for last-presented items in serial lists. Thirty lists of 10 numbers were categorized by recording half the list in a male voice and the other in a female voice. The order of the number was repeated every third list in one of the categories. Four groups of $S s(N=10)$ recalled the categories in serial or reverse order. The results indicated that repetition effects were isolated principally to the first-presented category.

The typical U-shaped serial position curve has been interpreted by Glanzer \& Cunitz (1966) in terms of a two-store model of memory. In this model items of information enter the memory system via a short-term memory (STM) mechanism and are encoded or transferred to a long-term memory (LTM) store. STM is viewed as a temporary holding mechanism of limited capacity. While new items entering STM displace old ones, LTM is seen as a relatively permanent store of information. In terms of the serial-position curve, items recalled from the beginning of a list were viewed as output from a LTM store while items at the end of a list were hypothesized to be a product of a STM mechanism.

An optimal recall strategy predicted by this model would be one which would first retrieve items from STM (last-presented items) and then items from LTM (first-presented items). Such a strategy has been reported for Ss allowed free recall of lists of words (Deese, 1957; Murdock, 1962). This model also offers a new interpretation of the results of a study by Posner (1964). Ss in this study recalled serially the last four (STM) digits of eight presented before recalling the first four (Reverse order of report), or were required to recall the eight digits serially. The Reverse condition showed superior recall, principally in the recall of the last four items. For Serial Ss, recall of the last four items was low and it appears that recall of the first items interfered with STM (last-items) recall.

A critical difference between the memory stores lies in the expected effects of repetition. Because information does not "accumulate" in the STM store but is displaced by new input or recall itself, learning would not be expected with the repetition of a given set of items. However, since LTM is hypothesized to retain information, repetition effects would be expected. Results consistent with this interpretation have been reported by Bartz (1968). In this study, patterned after the work of Hebb (1961), Ss serially recalled nine-digit strings where one string of digits was repeated at regular intervals. Irrelevant activity interpolated between list presentation and recall was found to reduce the recall of last-presented items (STM), but the rate at which the repeated string was learned was not different from that of Ss who recalled immediately. Serial position effects also showed that the learning of the repeated lists proceeded from the beginning to the end of the list.

The present experiment was conducted to investigate repetition effects where the repetition was limited to either the beginning or the end of a list. Serial lists of numbers were categorized into two half-spans by having a male speak one half-span and a female the other. The serial and reverse recall strategies (after Posner, 1964) were obtained by instructing Ss to report one voice prior to the other. Repetition was provided by repeating the sequence of items in one half-span in every third list. From a two-store model of memory significant improvement in recall was expected to be found in the recall of first half-spans (LTM) but not of second half-spans (STM). SUBJECTS

Eight groups of five Ss each were student volunteers from an undergraduate psychology course at lowa State University. They received extra course credit for their participation.

STIMULUS MATERIALS

The Ss listened to 30 lists of 10 digits each. The lists were composed of the digits 1 through 10 and were recorded at the rate of two digits per second. To form two subclasses within a list, a male spoke half of the digits and a female the other half. One half-span was repeated in every third list presented. The other half-span in these lists contained random orders of the five remaining digits. The lists without repetition were random orders of the 10 digits.

\section{RECORDING PROCEDURE}

Four tape recordings were prepared so that lists differed in terms of Position of Repetition (first or second half-span) and Voice (whether the first or second half-spans were spoken by a female voice). This procedure counterbalanced order of presentation of the half-spans and controlled for the possible differential recall of the digits spoken by the two voices.

\section{INSTRUCTIONS AND DESIGN}

Half of the Ss were instructed to report the male voice first and half were told to report the female voice first. These instructions resulted in half the
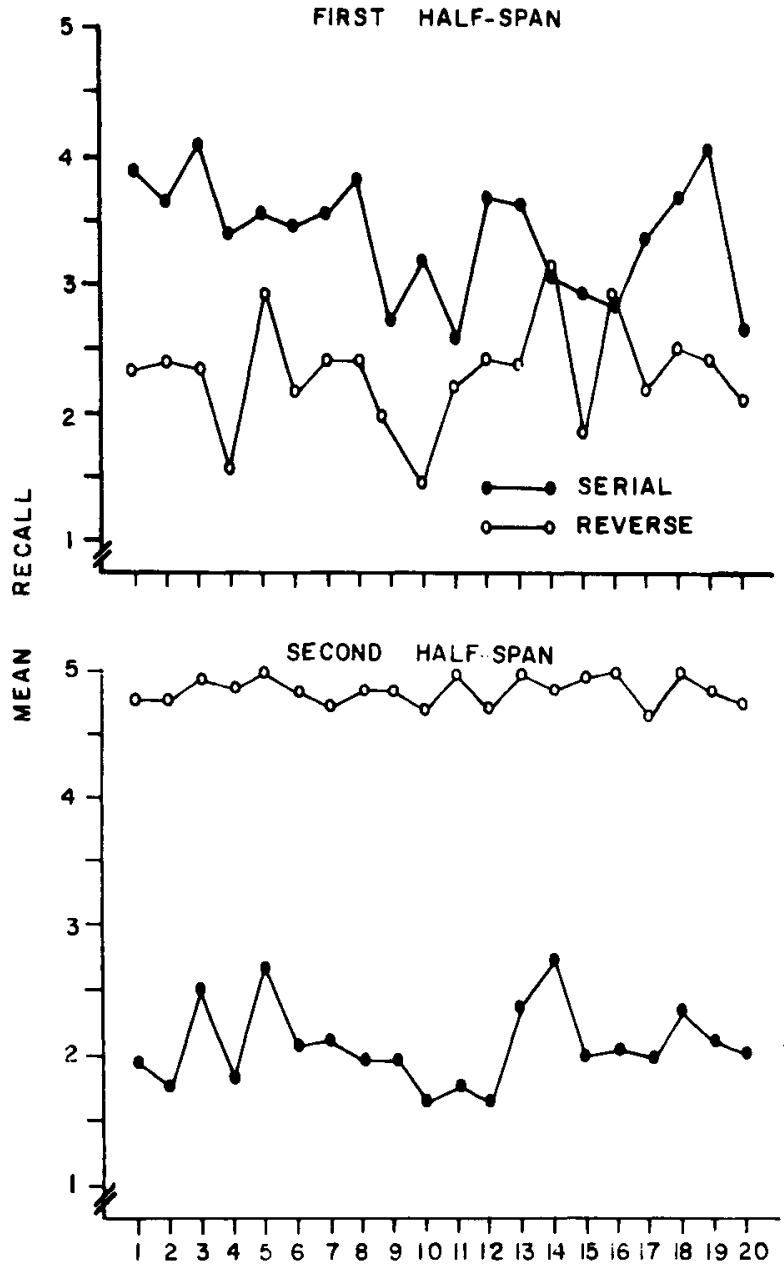

LISTS

Fig. 1. Mean recall on lists without repetition. 


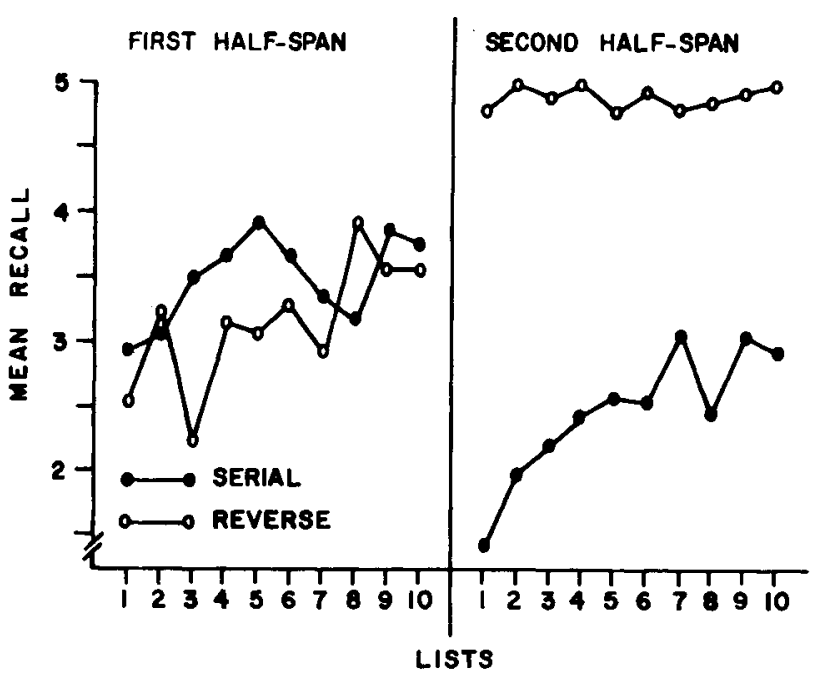

Fig. 2. Mean recall on lists with repetition.

Ss reporting the digits in serial order and half reporting the lists in reverse order (last half-spans first). By using each recording twice (once under each order of report), a $2^{4}$ by $n$ design was filled: Voice (whether the female voice spoke the first or second half-span) by Position of Repetition (repetition in the first or second half-span) by Order of Report (serial or reverse) by Half-Spans (first or second) by Lists (n lists: 10 with a repeated half-span and 20 with no repetition).

\section{PROCEDURE}

Each $S$ listened individually to one tape recording and recalled out loud immediately after each list. E recorded S's responses on a prepared scoring sheet.

\section{SCORING AND ANALYSIS}

The number of correctly recalled digits per half-span was determined by counting from the first digit recalled to the first error and then from the last digit recalled backwards to the first error on each half-span. Upon inspection, the differences attributable to Voice (male vs female) were negligible and the appropriate groups were combined in the analysis. Recall per half-span both in lists with repetition and those without was analyzed by means of analysis of variance. The Newman-Keuls procedure (Winer, 1962) was used to conduct tests among means.

\section{RESULTS}

Lists Without Repetition

The results are shown in Fig. 1. The expected Half-Spans by Order of Report interaction was obtained, $F(1,36)=369.84(p<.01)$. Serialorder-of-report Ss showed higher recall on first half-spans and reverse-order Ss higher, near maximum, recall on second half-spans. Moreover, in terms of overall recall, reverse order of report was superior to serial $\mathrm{F}(1,36)=11.42(\mathrm{p}<.01)$; and items in the second half-spans were recalled better than those in the first, $F(1,36)=36.96(p<.01)$.

In addition, Order of Report and Half-Spans interacted significantly with Lists, $F(19,684)=1.95(p<.01)$, and the Lists main effect was significant, $F(19,684=2.62(\mathrm{p}<.01)$. Inspection of Fig. 1 shows no consistent trends in recall over half-spans of the lists and the differences might best be attributed to variability in half-span difficulty.

Repetition Effects

Repetition effects were shown in three major ways: (1) in the general effects of repetition on half-span recall, (2) in the effects of repetition on patterns of recall over half-spans, and (3) in differences in patterns of recall within Orders of Report.

First, the presence of repetition significantly increased recall in the first half-spans but not in the second, $F(1,36)=13.03(p<.01)$. A clear separation of first half-spans as a function of repetition $(p<.01)$ was found, but the second half-spans did not differ significantly $(p>.05)$.
Second, first half-spans with repetition not only showed higher recall than those without, but also showed a consistent increase in recall over lists. In contrast, first half-spans without repetition showed greater variability in recall over lists and no consistent pattern of change. In second half-span recall, the effects of repetition were small with some gain in recall over lists being shown whether repetition was present or not. These significant effects were reflected in the interaction of Position of Repetition by Half-Spans by Lists, $F(9,324)=7.14,(p<.01)$.

The third major effect of repetition is shown in Fig. 2. This figure illustrates the significant differences in recall over half-spans of the lists with repetition under both orders of report, $F(9,324)=3.49(p<.01)$. Repetition effects were evident in both half-spans for Ss recalling serially, but only in the first half-spans for Ss under reverse recall instructions.

\section{DISCUSSION}

In general, the results of this experiment were consistent with the predictions of two-store models of memory. Ss employing the Reverse order of report, those supposedly "dumping" STM items prior to LTM retrieval, showed significantly greater recall than did Serial-recall Ss. Repetition effects were also found to be significant in first half-spans (LTM) but not in second half-spans (STM).

However, gains in recall were also found in the second half-span recall of Serial Ss. Recall of the second half-spans by Reverse Ss was near maximum and with little variability. It is possible that these results can be accounted for in terms of the information-processing strategies employed by Serial Ss and by Reverse Ss. The Serial Ss may have "concentrated" on first-presented items or, conversely, ignored last-presented items since they would be difficult to recall anyway. If this were the case, it is possible that there was little or no recall from a STM mechanism for Serial Ss and, consequently, this may be the reason that repetition effects were found in second half-spans. This strategy would also account for the higher first half-span recall found for Serial Ss. Possible differences in strategy of processing information might be experimentally controlled by employing both orders of report for all Ss and instructing the order of report with postlist cues. This procedure was employed by Murdock (1968) who found differences only in the recall of last-presented items (Serial recall less than Reverse).

It could also be argued that repetition effects were also evident in second half-span recall of Reverse Ss but that they could not be seen because recall was so very high. If stimulus materials were used which had a higher probability of error in recall, these repetition effects might be observed.

\section{REFERENCES}

BARTZ, W. H. Repetition and the memory stores. Journal of Experimental Psychology, in press.

GLANZER, M., \& CUNITZ, A. R. Two storage mechanisms in free recall. Journal of Verbal Learning \& Verbal Behavior, 1966, 5, 35 I-360.

HEBB, D. O. Distinctive features of learning in the higher animal. In J. B. Delafreshaye (Ed.), Brain mechanisms and learning. New York: Oxford University Press, 1961. Pp. 37-46.

MURDOCK, B. B., JR. The serial position effect of free recall. Journal of Experimental Psychology, 1962, 64, 482-488.

MURDOCK, B. B., JR. Serial order effects in short-term memory. Journal of Experimental Psychology: Monograph Supplement, 1968, 76, 1-15.

POSNER, M. I. Rate of presentation and order of recall in immediate memory. British Journal of Psychology, 1964, 55, 303-306.

WINER, B. J. Statistical principles in experimental design. New York: McGraw-Hill, 1962.

NOTE

1. This research was supported, in part, by PHS Research Grant MH 13192 from the National Institutes of Mental Health, Public Health Service. 